\title{
Hirayama Disease with Periscapular Involvement: A Rare Association
}

\author{
Vikram V. Holla Sanjeev Kumar Rakesh Shukla \\ Department of Neurology, King George's Medical University, Lucknow, India
}

\section{Key Words}

Hirayama disease $\cdot \mathrm{MRI} \cdot$ Anterior horn cell disorder

\begin{abstract}
Hirayama disease (HD) is a rare disease commonly seen in India and Japan typically presenting in adolescent age group with male predominance and asymmetrical distal pure motor, lower-motor neuron-type weakness. We report a patient of HD who had significant involvement of proximal periscapular muscles along with typical distal involvement. The patient also had scapular winging, which is rare in HD. He was treated conservatively with physiotherapy and hard cervical collar and is presently under follow-up during the static phase of disease.

(c) 2016 S. Karger AG, Basel
\end{abstract}

\section{Introduction}

Hirayama disease (HD) is a dynamic compressive ischemic cervical myelopathy, due to disproportionate growth between the vertebral column and dural sac. The natural course of disease is of limited duration (16.8-73.2 months), that is, it is a self-limited disease [1]. Classically,
HD occurs in adolescent males with insidious onset unilateral upper limb distal weakness and muscle atrophy with variable periods of progression and spontaneous arrest. The disease progression is halted by early diagnosis and conservative management with cervical collar. Bilateral involvement in $\mathrm{HD}$ is considered to be a severe variant [2].

\section{Case Presentation}

A 20-year-old male presented with atrophy and weakness of both upper limbs for the last 1 year. He noticed upper limb distal muscle atrophy in the left side followed by the right side at an interval of 1 month. The atrophy of both the upper limbs progressed over 4-5 months and involved shoulder and scapular region also. The weakness also started in a similar fashion first in the left upper limb followed by right upper limb. He found difficulty in gripping objects, holding a glass of water and in writing. The weakness gradually involved proximal shoulder and scapular region muscles also. He was unable to lift heavy objects overhead as well as found it difficult to dress himself and comb his hair. There was no weakness in the lower extremities. He also did not have sensory and bowel/bladder complaints. He denied trauma to neck, neck pain or restriction of movement in the neck. The family history was negative for similar complaints.

On examination, the patient was moderately built and nourished with a height of $183 \mathrm{~cm}$, neck length of $14 \mathrm{~cm}$ and neck-

\section{KARGER}

E-Mail karger@karger.com

www.karger.com/aon
(C) 2016 S. Karger AG, Basel

0972-7531/16/0231-0056\$39.50/0
Prof. Rakesh Shukla

Department of Neurology

King George's Medical University

Lucknow, Uttar Pradesh 226003 (India)

E-Mail rakeshshukla_rakesh@ rediffmail.com 
Fig. 1. Image showing asymmetrical scapular winging (black arrow), atrophy of shoulder muscles, forearm muscles (white arrow head) and small muscles of hand (black arrow head).

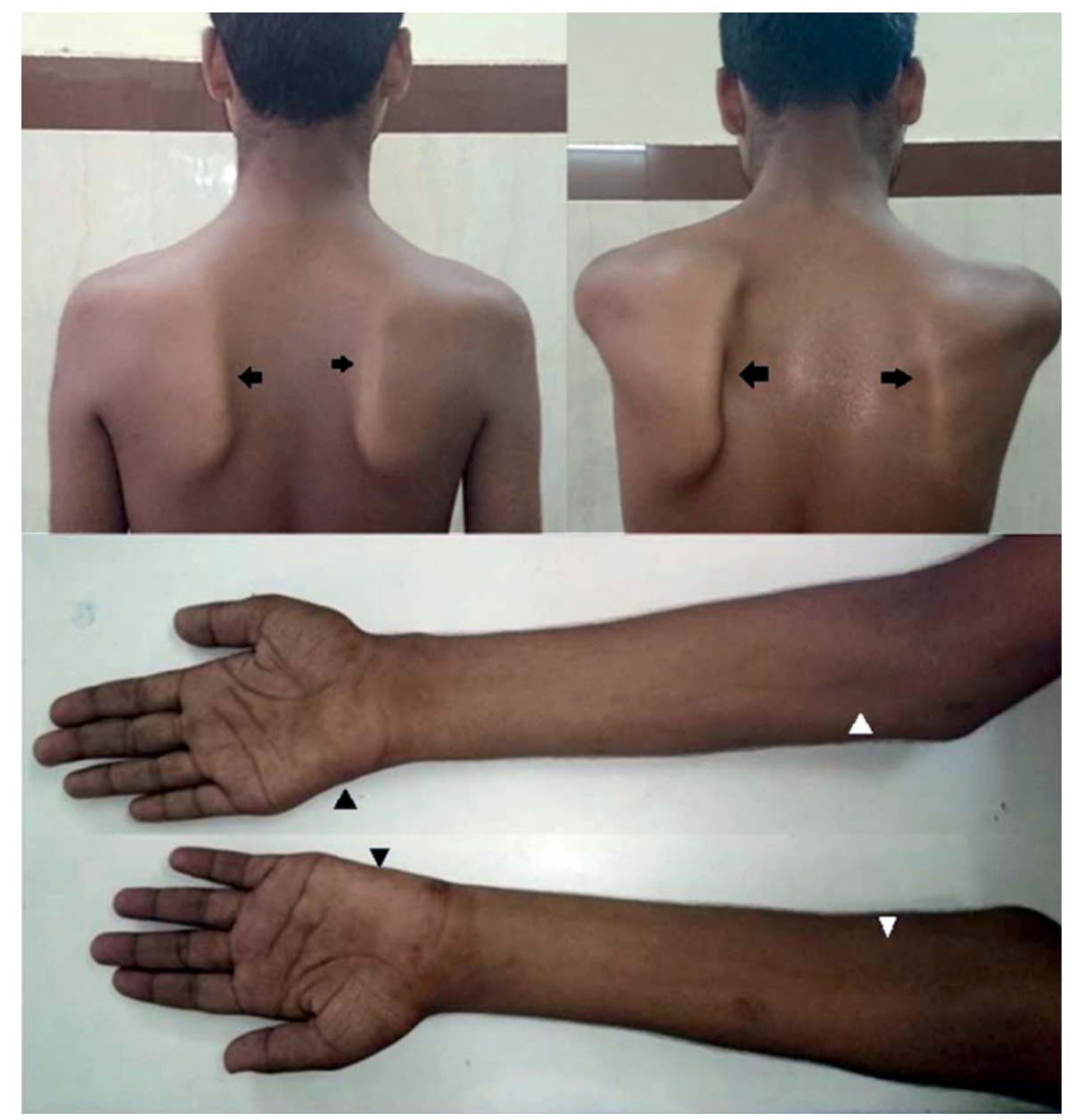

height ratio of 13.1. Higher mental function and cranial nerve examination were normal. Motor system examination revealed atrophy of rhomboids, supra and infraspinatus, serratus anterior, pectoralis, muscles of arms, forearm and small muscles of hands. Atrophy was more in the left side compared to right side and there was relative sparing of brachioradialis on both sides (fig. 1). Tone was decreased at all joints of both upper limbs. Power assessment revealed MRC grade 2-3 across all joints in both upper limbs. Scapular winging was evident more on left side. Biceps jerk was 1+ with absent triceps and supinator jerks on both sides.

Polyminimyoclonus was seen in fingers of both hands. Motor system examination was completely normal in lower limbs with normal flexor plantar response. Sensory system examination was also normal. There was no evidence of any cerebellar or extrapyramidal system impairment. Systemic examination was also normal.

His biochemical and hematological parameters were normal. Nerve conduction study (NCS) revealed normal sensory nerve action potentials (SNAPs) in bilateral median, ulnar and radial nerves with reduced compound muscle action potentials in bilateral supraspinatus, infraspinatus, biceps, adductor digiti minimi $(\mathrm{ADM})$ and abductor pollicis brevis (APB). Electromyographic (EMG) study found large amplitude polyphasic muscle unit action potentials with reduced recruitment and spontaneous activity in the form of fibrillation and fasciculations in bilateral biceps, triceps, ADM, APB and rhomboids. NCS and EMG studies were normal in lower limbs. They suggested the possibility of anterior horn cell disorder confined to cervical distribution with no evidence of any conduction block. MRI of the cervical spine with dynamic flexion study with gadolinium contrast was done revealing findings suggestive of HD (fig. 2). He was managed conservatively with neck immobilization by hard cervical collar and physiotherapy. Patient was kept under regular follow-up. The patient's symptoms have been static during the 6-month follow-up.

\section{Discussion}

$\mathrm{HD}$, also called juvenile muscular atrophy or monomelic amyotrophy of upper limb, is an uncommon disease often seen in India and Japan [1]. Typically, it presents in adolescent age group with male predominance and asymmetrical distal pure motor, lower-motor neuron-type weakness. Usually, it progresses over 3-5 years, 


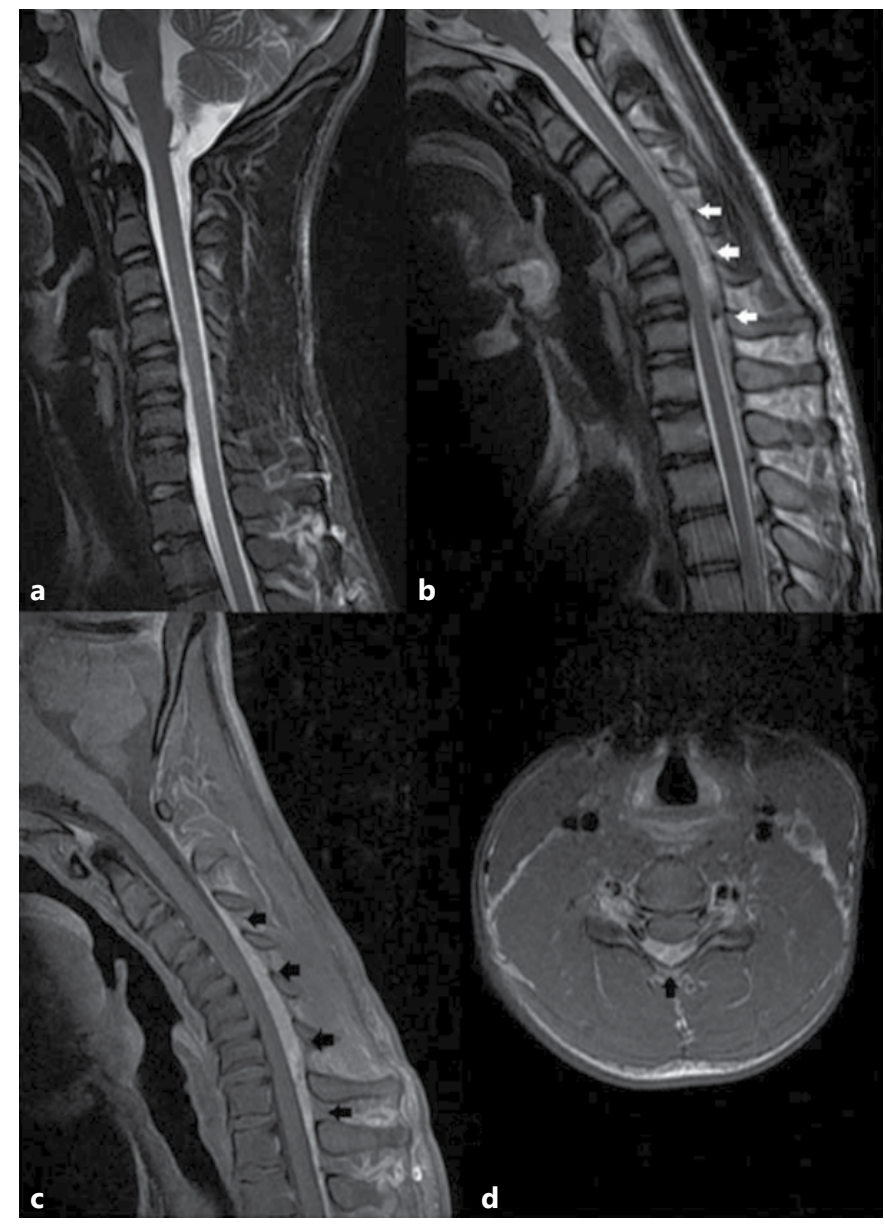

Fig. 2. MRI of cervical spine. a T2-weighted image showing loss of cervical lordosis in neutral position. b Apposition of cervical spinal cord from C3 to D1 with displacement of posterior dural sheath (white arrow) in sagittal flexion T2-weighted image. c and d Posterior epidural venous congestion (black arrow) in post-contrast T1-weighted sagittal and axial images.

thereafter becoming static unlike other motor neuron diseases that are usually relentlessly progressing. The most commonly affected segment is C7-T1 with some spill over to C6 level. Although typically HD is asymmetrical in onset and severity, it has been observed to be symmetrical in up to $10 \%$ of cases as reported by Pradhan [2]. Rarely HD can involve more proximal muscles of shoulder and arm alone or along with distal muscles [3, 4]. Most of such cases are unilateral. In our case, the patient had weakness and atrophy starting from distal muscles and extending to proximal muscles up to periscapular muscles. Also, he had severe involvement of periscapular muscles with prominent scapular winging, which is very rare for HD. The most commonly proposed pathophysiology for $\mathrm{HD}$ is the anterior displacement of the spinal cord and dural sac causing apposition of cord over the disc and posterior spinal ligament. This vascular insufficiency leads to ischemic changes in most vulnerable anterior horn cells leading to symptomatology of HD. Repeated injury to cervical anterior horn cells leads to spinal cord atrophy. The reason for the anterior dural sac displacement is said to be due to difference in the rate of elongation of dura and the vertebral column. During the growth spurt in adolescence, the vertebral column elongates more compared to the dural sac, thereby stretching and tightening and thus weakening the normal attachment between the dura and vertebral column. This tightening increases during flexion, and hence, the dura get further separated from vertebral column and displaces forward.

The differential diagnosis that has to be considered in a typical distal presentation of HD is radiculopathy and neuropathy. Among radiculopathy, compressive radiculopathy can present with unilateral or asymmetrical involvement affecting C8-T1 roots with absence of sensory loss due to overlap by adjacent roots, and NCS can have normal SNAP strongly mimicking HD. But the positive sensory symptoms like parasthesia and radicular pain help in differentiating. Lower cord brachial plexopathy can also present similarly but sensory loss and absence of SNAPs in NCSs is common. Among neuropathies, multifocal motor neuropathies with conduction blocks (MMNCB) and lead neuropathy can present as pure motor upper limb predominant distal onset asymmetrical weakness. While finding of conduction block and demyelinating pattern in NCS help differentiate MMNCB, occupational history of lead exposure and raised serum lead levels help in diagnosing lead neuropathy. Syringomyelia can sometime present without typical dissociative sensory loss and hence present similar to HD but usually will be symmetrical and MRI can differentiate the two.

MRI cervical spine plays the most crucial role in diagnosis of HD especially dynamic flexion extension with contrast. MRI cervical spine may show cord atrophy at lower cervical level. Occasionally, there may be very subthe cord atrophy or no atrophy at all there by leading to false negative results. Flexion imaging of MRI cervical spine typically shows forward displacement of the posterior dural wall and a well-enhanced crescent-shaped mass in the posterior epidural space of the lower cervical canal which disappears once the neck returns to a neutral position [5]. MRI cervical spine revealed the detached posterior ligament from $\mathrm{C} 3$ to $\mathrm{T} 1$ vertebrae and maximal compressive effect from $\mathrm{C} 4$ to $\mathrm{C} 8$ spine (fig. 2), which explained the peculiarity of findings in our case. There is 
arrest of progression of disease after the initial period of progression. Since HD is a flexion-induced injury to the cervical cord, immobilization with hard collar halts the disease progression. Most of the cases either become static or may improve after wearing the collar. Duroplasty is advised in patients who have progression of disease even after conservative treatment. Although there are various surgical procedures aimed at correcting dural tightening, the results are inconclusive as the number of cases is limited.

\section{Conclusion}

Thus, it is very important to consider HD even when the disease is symmetrical or involves the proximal muscles of arm and scapula. In clinical practice, a patient presenting with symptomatology suggesting motor neuron disease, a diagnosis of HD gives the patient a good prognosis compared to other forms of motor neuron disease. The diagnosis can be confirmed by MRI, and it is important to request for dynamic flexion/ extension images as the findings can be missed in neutral position.

\section{References}

1 Gourie-Devi M, Nalini A: Long-term followup of 44 patients with brachial monomelic amyotrophy. Acta Neurol Scand 2003;107: 215-220.

2 Pradhan S: Bilaterally symmetric form of Hirayama disease. Neurology 2009;72:20832089.

\section{Authorship Contribution}

Drs. V.V. Holla and S. Kumar: drafting and finalizing the manuscript and editing the image. Prof. R. Shukla: editing and finalizing the manuscript.

\section{Source of Support in the Form of Grants, Equipment, Drugs}

None.

\section{Disclosure Statement}

None.

\section{Acknowledgment}

Nil.

\section{Written Consent from Patient}

Taken.
3 Neves MA, Freitas MR, Mello MP, Dumard $\mathrm{CH}$, Freitas GR, Nascimento OJ: Benign monomelic amyotrophy with proximal upper limb involvement: case report. Arq Neuropsiquiatr 2007;65:524-527.
4 Yaguchi H, Takahashi I, Tashiro J, Tsuji S, Yabe I, Sasaki H: Scapular winging as a symptom of cervical flexion myelopathy. Intern Med 2007;46:511-513.

5 Pradhan S, Gupta RK: Magnetic resonance imaging in juvenile asymmetric segmental spinal muscular atrophy. J Neurol Sci 1997; 146:133-138. 